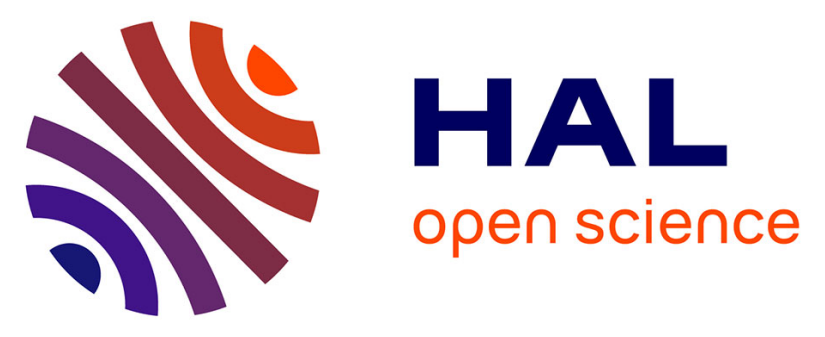

\title{
Mesure simultanée d'une température moyenne et d'une différence de température centrée sur cette moyenne. application à l'enregistrement direct du pouvoir thermoélectrique en fonction de la température
} J.P. Troadec, Dang Tran Quan, Y. Colin

\section{To cite this version:}

J.P. Troadec, Dang Tran Quan, Y. Colin. Mesure simultanée d'une température moyenne et d'une différence de température centrée sur cette moyenne. application à l'enregistrement direct du pouvoir thermoélectrique en fonction de la température. Revue de Physique Appliquée, 1977, 12 (3), pp.503509. 10.1051/rphysap:01977001203050300 . jpa-00244203

\section{HAL Id: jpa-00244203 \\ https://hal.science/jpa-00244203}

Submitted on 1 Jan 1977

HAL is a multi-disciplinary open access archive for the deposit and dissemination of scientific research documents, whether they are published or not. The documents may come from teaching and research institutions in France or abroad, or from public or private research centers.
L'archive ouverte pluridisciplinaire HAL, est destinée au dépôt et à la diffusion de documents scientifiques de niveau recherche, publiés ou non, émanant des établissements d'enseignement et de recherche français ou étrangers, des laboratoires publics ou privés. 
MESURE SIMULTANÉE D'UNE TEMPÉRATURE MOYENNE ET D'UNE DIFFÉRENCE DE TEMPÉRATURE CENTRÉE SUR CETTE MOYENNE. APPLICATION A L'ENREGISTREMENT DIRECT DU POUVOIR
THERMOÉLECTRIQUE EN FONCTION DE LA TEMPÉRATURE

\author{
J. P. TROADEC, DANG TRAN QUAN et Y. COLIN \\ Groupe d'Electronique et de Physique des Matériaux, Université de Rennes. U. E. R. «S. P. M.», \\ 35031 Rennes, Cedex, France
}

(Reçu le 9 juin 1976, accepté le 6 décembre 1976)

\begin{abstract}
Résumé. - Nous décrivons un appareil analogique permettant de déterminer la moyenne et la différence de températures thermodynamiques à partir de signaux délivrés par deux thermocouples. Nous envisageons l'application de ce dispositif à l'enregistrement du pouvoir thermoélectrique en fonction de la température.
\end{abstract}

\begin{abstract}
An analog apparatus to determine the average and the difference of the thermodynamic temperatures given by two thermocouples is described. It is designed to record the temperature dependence of the thermoelectric power.
\end{abstract}

Introduction. - La mesure précise du pouvoir thermoélectrique point par point en fonction de la température est une opération laborieuse qui, outre des précautions expérimentales particulières, réclame de longues heures d'exploitation d'enregistrements.

Nous avons cherché à mettre au point un appareillage simple, aussi peu coûteux que possible, permettant l'enregistrement direct du pouvoir thermoélectrique avec une précision suffisante, en vue d'une première approche du phénomène pour un matériau nouveau ou de contrôles sur des matériaux ou des composants connus.

Deux appareils ont été construits avec des composants courants et leurs indications ont été comparées à des mesures ponctuelles de pouvoir thermoélectrique effectuées à l'aide de la méthode imaginée par Conan [1] à partir des principes posés et utilisés par Caskey et coll. [2].

Dans la première partie de notre exposé, nous indiquerons les principes qui ont déterminé la construction des prototypes réalisés. Puis nous entrerons dans les détails de la réalisation, étage par étage. Enfin après une analyse rapide de la précision, nous donnerons des résultats expérimentaux de mesures de pouvoir thermoélectrique effectuées sur du nickel, qui nous servira d'étalon, et sur des composés nouveaux.

1. Principe de l'appareil (Fig. 1). -1.1 Mesure DE DIFFÉRENCE DE TEMPÉRATURE. - Afin de ne pas court-circuiter l'échantillon $M$ soumis à un test de pouvoir thermoélectrique, la température de ses extrémités est détectée par deux thermocouples indépendants, aussi identiques que possible, délivrant respectivement les tensions $e_{1}$ et $e_{2}$. Pour que ces signaux soient traités exactement dans les mêmes conditions, ils sont commutés périodiquement, à tour de rôle, sur l'entrée d'un même amplificateur $A_{1}$ suivi d'un linéariseur destiné à compenser la non-linéarité de la caractéristique température-tension des thermocouples.

La sortie du linéariseur se compose alors d'une tension continue, image de la moyenne des températures captées par les deux thermocouples, et d'un signal carré, dont la valeur, crête à crête, est proportionnelle à la différence de ces températures.

Il s'ensuit que l'appareil permettra toutes mesures de différence de température, qu'il sera utilisable aussi bien pour faire de l'ATD que des études de pouvoir thermoélectrique ou de conductivité thermique et qu'il pourra servir de capteur-transmetteur de mesure pour des régulations de différence de température. Il est en effet facile de séparer par un condensateur les deux signaux superposés à la sortie du linéariseur ; un étage redresseur fournira ensuite une tension continue proportionnelle à la différence des températures.

Pour des enregistrements en fonction de la température, il sera commode d'utiliser la valeur moyenne $T$ du signal délivré par le linéariseur. 


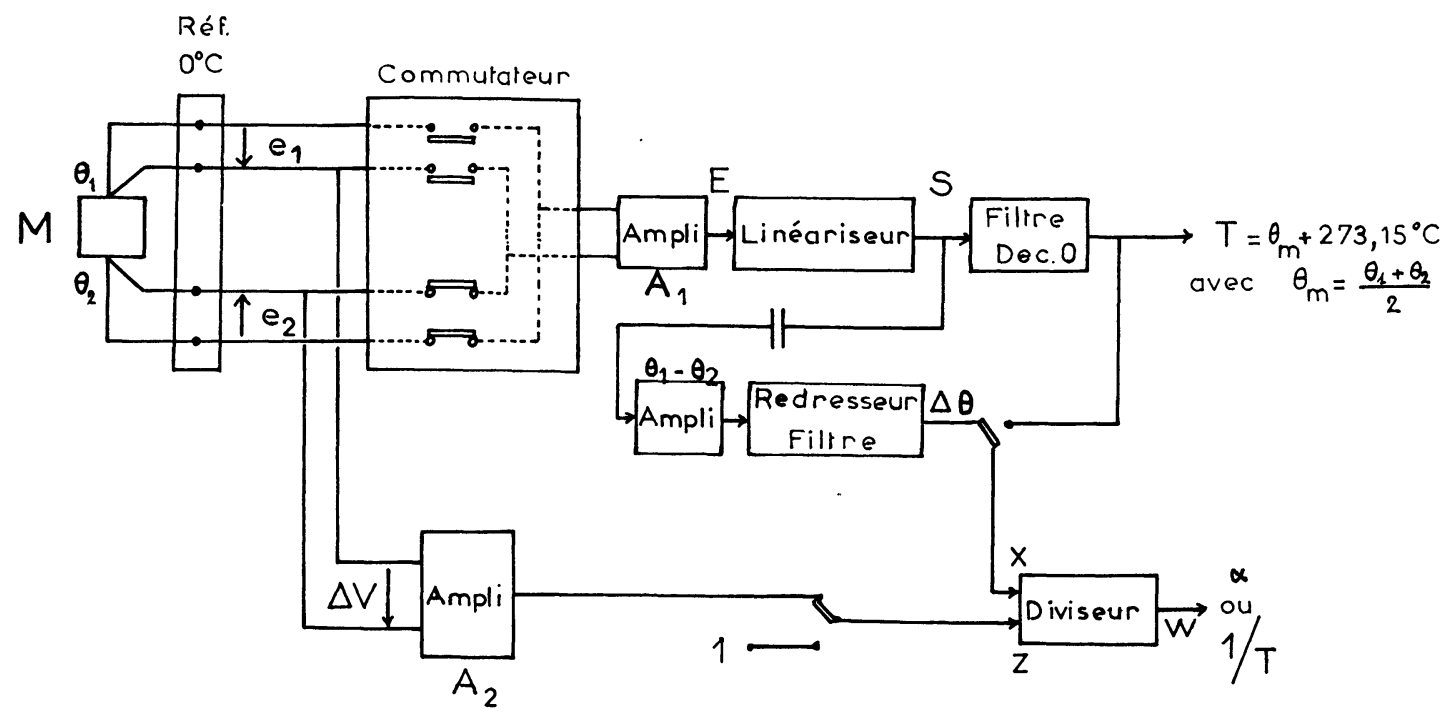

Fig. 1. - Schéma synoptique de l'appareillage.

1.2 Mesure De tension theRMoÉLECTRIQUe. - La différence de potentiel thermoélectrique $\Delta V$ peut être captée entre deux fils de même nature des thermocouples utilisés. Elle est alors amplifiée $\left(A_{2}\right)$, puis envoyée à l'entrée $Z$ d'un diviseur analogique tandis que la tension proportionnelle au gradient thermique parvient à l'autre entrée $\mathrm{X}$. On obtient donc à la sortie du diviseur le pouvoir thermoélectrique $\alpha$ de l'échantillon par rapport au conducteur choisi.

La mesure peut être effectuée, soit en régime thermique stationnaire, soit en remontée lente de température, de l'ordre du degré par minute. Elle est basée sur l'approximation $\alpha=\Delta V / \Delta \theta$, la différence de température, éventuellement régulée, étant comprise entre 5 et $15^{\circ} \mathrm{C}$.

1.3 INVERSE DE LA TEMPÉRATURE THERMODYNAMIQUE. - Comme le montage peut en outre constituer l'un des éléments d'un dispositif de mesure de conductivité électrique, nous avons prévu d'élaborer un signal $1 / T$ proportionnel à l'inverse de la température thermodynamique moyenne de l'échantillon étudié.

2. Etude des différents étages. - Les performances de l'appareillage dépendent de celles des circuits et des composants utilisés. Désireux de limiter le coût de revient, nous avons choisi de réaliser les prototypes avec du matériel courant, en employant systématiquement des amplificateurs opérationnels, en raison des qualités propres de ces dispositifs : gain en tension élevé, grande impédance d'entrée, faible impédance de sortie et faible dérive, facilité de réglage du gain indépendamment des caractéristiques propres de l'amplificateur grâce à un réseau de contre-réaction approprié.

2.1 Amplification et COMMUTATION DES SIGNAUX DE TEMPÉRATURE (Fig. 2). - La plupart des thermocouples courants délivrent, pour une soudure froide à

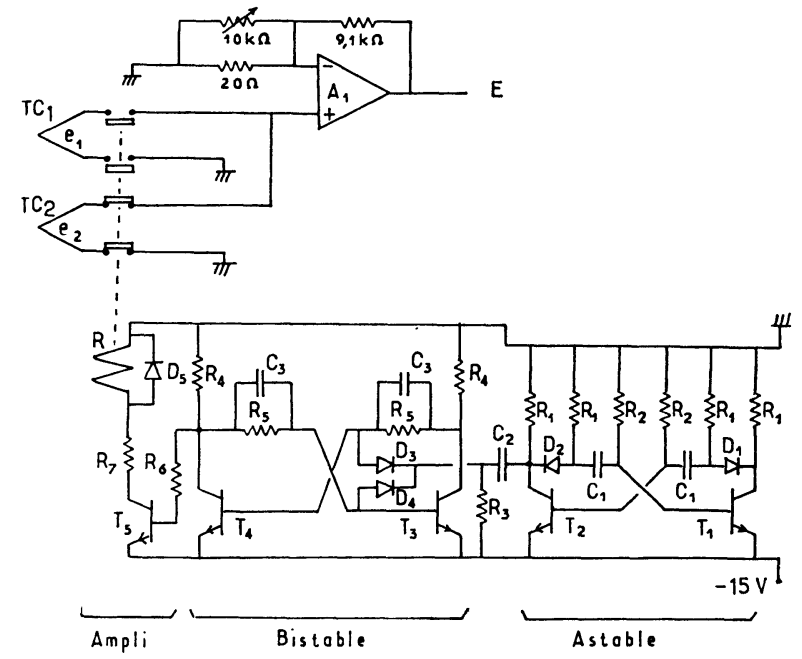

Fig. 2. - Amplification et commutation des tensions fournies par les thermocouples:

$T_{1}$ à $T_{5}:$ TIS $98 \quad D_{1}$ à $D_{4}: 1 \mathrm{~N} 914 \quad D_{5}:$ OA 95

$\mathrm{TC}_{1}$ à $\mathrm{TC}_{2}:$ thermocouples

$\mathbf{A}_{1}$ : amplificateur TA 20

$\begin{array}{llll}R_{1}=20 \mathrm{k} \Omega & R_{2}=200 \mathrm{k} \Omega & R_{3}=100 \mathrm{k} \Omega & R_{4}=1,8 \mathrm{k} \Omega \\ R_{5}=10 \mathrm{k} \Omega & R_{6}=10 \mathrm{k} \Omega & R_{7}=470 \Omega & \\ C_{1}=0,68 \mu \mathrm{F} & C_{2}=1 \mathrm{nF} & C_{4}=1 \mathrm{nF} .\end{array}$

$0{ }^{\circ} \mathrm{C}$, des tensions maximales utilisables $e_{\max }$ comprises entre 18 et $55 \mathrm{mV}$.

Comme il est souhaitable, d'une part pour minimiser l'effet de la dérive, d'autre part pour attaquer le linéariseur dans les meilleures conditions, de réaliser l'amplification maximale au niveau du premier étage et comme la tension admissible à la sortie d'un amplificateur classique est de $10 \mathrm{~V}$, nous avons été amenés à choisir pour le premier étage un gain

$$
A_{1}=10 / e_{\max }
$$

voisin de 480 pour le couple cuivre-constantan, auquel, 
pour fixer les idées, nous nous réfèrerons habituellement dans la suite.

Afin d'obtenir ce gain relativement élevé avec une dérive minimale, nous avons utilisé un amplificateur en montage non inverseur avec des résistances de bouclage de faibles valeurs; cet amplificateur présente un fort taux de réjection de mode commun et une faible dérive en tension; comme la résistance des thermocouples est peu importante, un courant de polarisation relativement élevé (ici < $70 \mathrm{nA}$ ) est admissible.

Pour présenter alternativement les tensions délivrées par les deux thermocouples à l'entrée de cet amplificateur, nous avons réalisé un commutateur analogique.

Dans une première version nous avons utilisé un montage à transistors à effet de champ. Malheureusement, du fait du courant de fuite trop important que présentent ces éléments lorsqu'ils sont bloqués, cette solution ne garantit une bonne indépendance des mesures de température et de tension thermoélectrique que si la résistance de l'échantillon à tester n'est pas trop élevée.

Une solution moins restrictive consiste à utiliser un relais électromécanique : nous l'avons réalisée en attaquant un relais à deux contacts inverseurs par une tension carrée à une fréquence voisine de $5 \mathrm{~Hz}$ en veillant à obtenir deux alternances rigoureusement identiques afin de conserver la symétrie des mesures et de faciliter ainsi la séparation ultérieure du signal de différence et du signal de température moyenne.

Le signal de commutation est fourni par un multivibrateur astable travaillant à une fréquence de $10 \mathrm{~Hz}$, suivi d'une bascule bistable, utilisée en diviseur par deux et en égaliseur des deux alternances, et d'un amplificateur destiné à fournir au relais un courant suffisant pour le faire basculer (Fig. 2).

2.2 LINÉARISEUR. - La caractéristique tension de sortie-température du premier étage est évidemment analogue à la caractéristique tension-température du thermocouple utilisé, elle est non linéaire.

Pour disposer d'une tension proportionnelle à la température $\theta$ mesurée, il est nécessaire d'introduire dans la chaîne de traitement du signal un organe correcteur présentant une non-linéarité exactement inverse de celle du capteur.

Parmi les techniques actuellement disponibles, celle du linéariseur à diodes est la moins coûteuse.

Si l'étage d'entrée délivre une tension $E=g(\theta)$, nous devons avoir en sortie du linéariseur

$$
S=k g^{-1}(E)=k g^{-1}[g(\theta)]=k \theta \text {. }
$$

La caractéristique $S(E)$ étant ainsi fixée, il est commode de prendre comme origine des tensions le point correspondant à $0^{\circ} \mathrm{C}$ : cette température est facile à obtenir et ce choix permet de disposer d'une échelle de tensions suffisamment étendue pour la réalisation du linéariseur.

Dans un domaine de tension restreint, la courbe $S(E)$ est approchée par un segment de droite, qui est la caractéristique entrée-sortie, dans ce domaine, d'un amplificateur opérationnel. Pour approcher l'ensemble de la courbe, il suffit de modifier cette caractéristique, par exemple en changeant la résistance de contreréaction de l'amplificateur, par addition ou suppression de résistances en parallèle à l'aide de diodes devenant passantes au-delà d'un certain seuil de tension.

La figure 3 montre le linéariseur que nous avons étudié pour le couple cuivre-constantan $(-200$ à $\left.+400^{\circ} \mathrm{C}\right)$. Nous avons limité le nombre de diodes à 14 : la précision théorique serait évidemment meilleure si l'on approchait la courbe $S(E)$ par un nombre plus élevé de segments, mais en pratique la dérive globale de l'étage, liée aux variations des tensions de seuil et courants inverses des diodes, est d'autant plus importante que le nombre de diodes est plus grand.

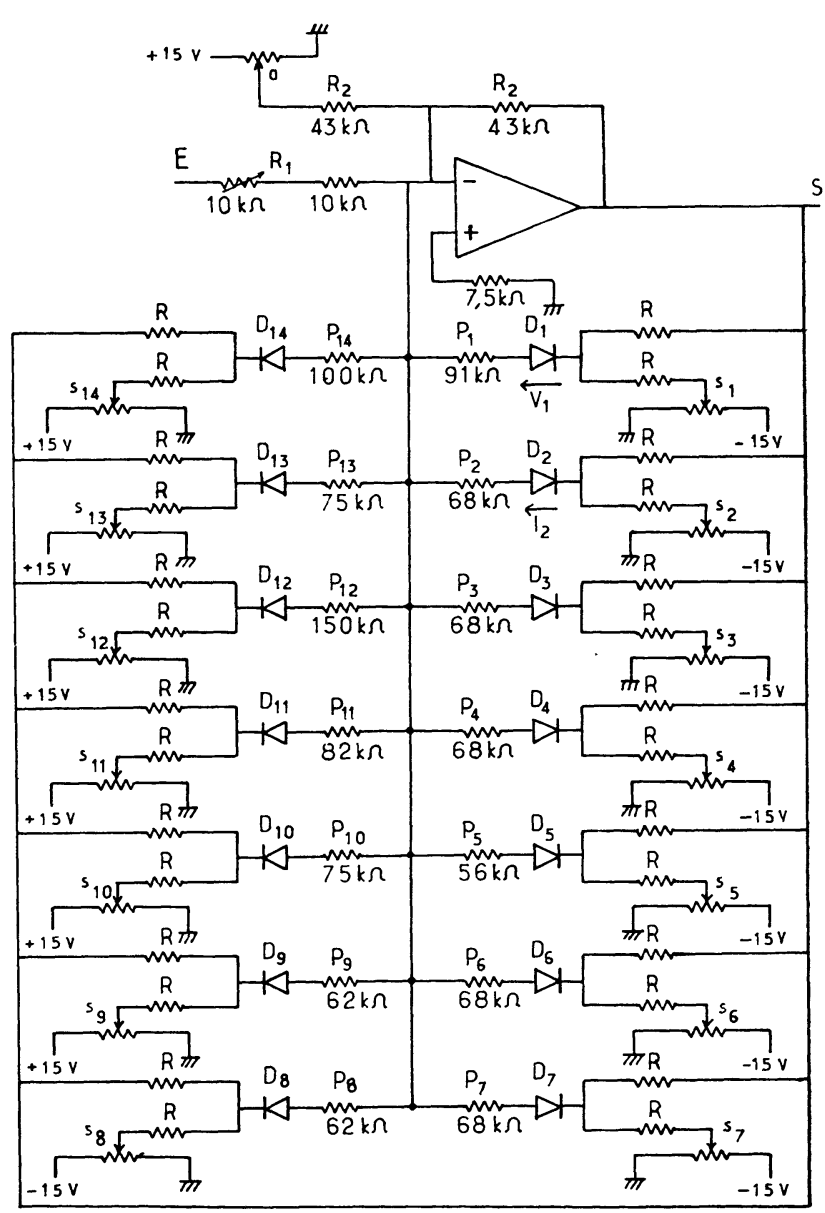

$\mathrm{R}=100 \mathrm{k} \Omega$

Fig. 3. - Schéma du linéariseur cuivre-constantan.

2.3 SÉPARATEUR DE SIGNAUX : DIFFÉRENCE DE TEMPÉRATURE. TEMPÉRATURE MOYENNE. - Le signal appliqué à l'entrée du linéariseur est en fait celui qui provient de la commutation périodique des thermocouples à l'entrée du premier étage : nous disposons donc à la sortie du linéariseur d'une tension moyenne, proportionnelle à la moyenne $\theta_{M}$ des températures captées, à 


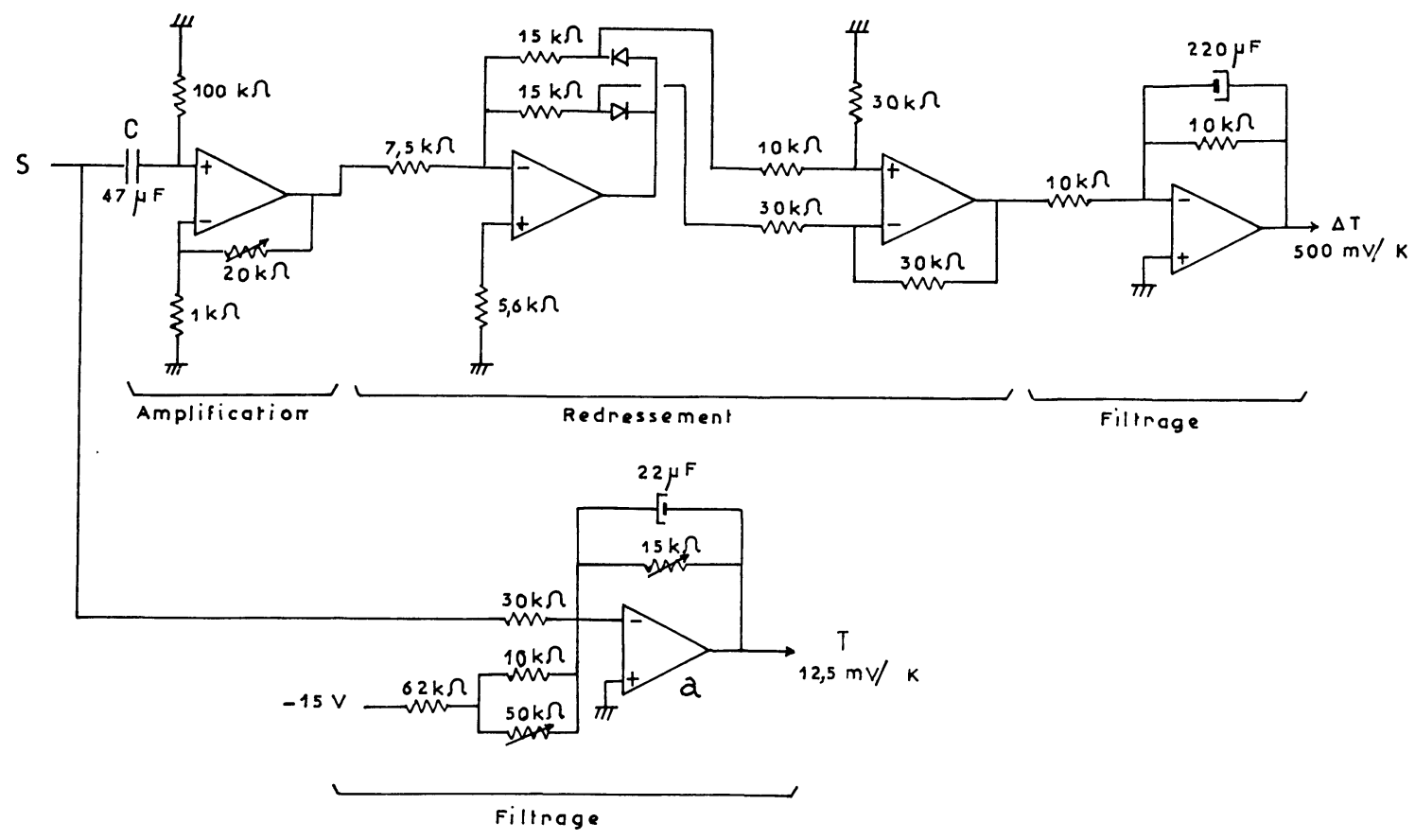

FIG. 4. - Séparation des signaux.

laquelle se trouve superposé un signal carré dont l'amplitude est proportionnelle à la différence de ces températures.

Il convient de séparer les deux composantes de ce signal.

2.3.1 Différence de température (Fig. 4). - Le signal carré est isolé par un condensateur, qui doit avoir une capacité élevée pour tenir compte de la faible fréquence de travail ; il est ensuite amplifié 20 fois et transmis à un étage redresseur ultralinéaire double alternance, qui procure un gain supplémentaire de 2 .

Nous disposons alors d'un signal continu, toujours positif, représentant la différence de température avec un coefficient de proportionnalité élevé $\left(500 \mathrm{mV} /{ }^{\circ} \mathrm{C}\right.$ pour un couple cuivre-constantan, avec $C=47 \mu \mathrm{F})$. Le gain de l'étage peut être réglé à $10^{-3}$ près.

En fait ce signal se trouve perturbé par les pics de commutation et un filtrage s'avère nécessaire : celui-ci procure en outre l'avantage d'inverser le signal et de le présenter avec une phase correcte à l'entrée du diviseur chargé de réaliser l'opération $\Delta V / \Delta \theta$, lorsque l'appareil est utilisé en mesureur de pouvoir thermoélectrique.

Le pouvoir séparateur est limité par la détection des tensions parasites (notamment de fréquence $50 \mathrm{~Hz}$ ) superposées au signal utile; un câblage judicieux des masses permet la mesure de différences de température à partir de $0,5^{\circ} \mathrm{C}$.

\subsubsection{Température thermodynamique moyenne} (Fig. 4). - Un simple filtrage et un décalage de l'origine fournissent, à partir de la sortie $\mathrm{S}$ du linéariseur, une tension $T$ proportionnelle à la moyenne des températures thermodynamiques mesurées.

La précision de cet étage est essentiellement liée à l'ajustement du gain $a$, qui peut être meilleur que $10^{-3}$. Dans le cas de la réalisation pour thermocouples cuivre-constantan, nous nous sommes arrangés pour que le coefficient de proportionnalité soit de $12,5 \mathrm{mV} / \mathrm{K}$.

Pour obtenir $1 / T$, nous utilisons un diviseur analogique (428J Analog Devices) qui délivre un signal de sortie

$$
W=10 \frac{Z}{X}
$$

proportionnel au rapport des tensions $Z$ et $X$ appliquées à ses entrées.

Un amplificateur additionnel de gain 1,2 permet d'envoyer sur l'entrée X le signal $T$ avec une phase correcte. $Z$ est fixé de façon à obtenir, en sortie $W$, $800 / T \mathrm{~K}(Z=1,2 \mathrm{~V} ;|Z / X|<1)$ (Fig. 5).

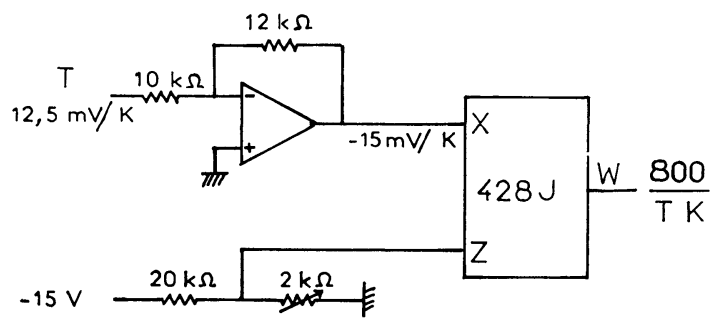

Fig. 5. - Obtention d'une tension proportionnelle à l'inverse de la température thermodynamique moyenne.

2.4 Pouvoir thermoÉleCtriQue. - Lorsqu'on utilise le montage en mesureur de pouvoir thermoélectrique, les bornes de l'échantillon se trouvent référencées à la masse dans le dispositif de mesure des 
températures : la mesure du pouvoir thermoélectrique ne peut alors s'effectuer qu'à l'aide d'une méthode différentielle (Fig. 6). L'échantillon $\mathrm{M}$ de résistance $R$ se trouve alors traversé par un courant $I$ qui est le courant d'entrée de l'amplificateur de mesure ; la tension réelle à ses bornes serait donc

$$
\Delta V_{1}=\Delta V+R I
$$

si ne venaient s'y ajouter des tensions parasites dues à la dérive $i$ du courant d'entrée et la dérive $e_{\mathrm{d}}$ de la tension d'offset ramenée à l'entrée de l'amplificateur.

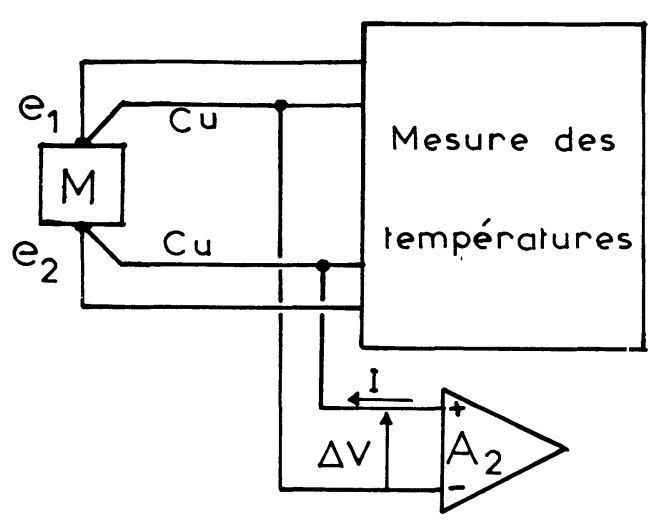

Fig. 6. - Principe de la mesure de la tension thermoélectrique.

Il en résulte que pour effectuer, dans de bonnes conditions (erreurs systématiques faibles), des mesures de pouvoir thermoélectrique sur des échantillons de résistance $R$ élevée, il est nécessaire de choisir un amplificateur présentant une impédance d'entrée différentielle très élevée, un courant $I$ d'entrée très faible et des dérives négligeables; c'est-à-dire un système ayant pour base un amplificateur très performant, donc coûteux.

Pour obtenir une impédance d'entrée différentielle très grande nous avons été amenés à utiliser un montage mettant en œuvre deux amplificateurs identiques suivis d'un étage différentiel de gain unité, dont le rôle est de délivrer une tension référencée à la masse (Fig. 7). Ce montage permet d'assurer une bonne indépendance de la mesure de tension thermoélectrique et de la mesure des températures.

Afin de minimiser l'effet de courant de polarisation et de sa dérive, nous avons utilisé des amplificateurs dont l'étage d'entrée est constitué par des transistors à effet

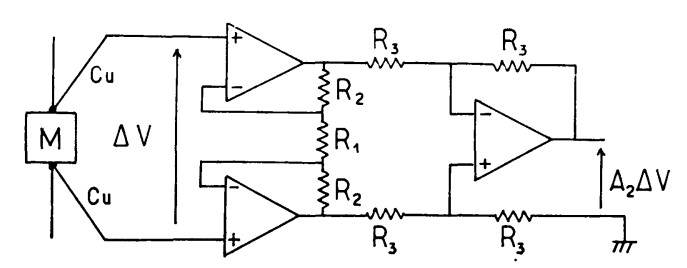

Fig. 7. - Amplification de la tension thermoélectrique. de champ à jonction, dont la fuite de grille se réduit à quelques dizaines de picoampères (TB $62 \mathrm{~B}$ de TE).

L'amplificateur différentiel de sortie n'influence pratiquement pas la précision de la mesure (nous avons choisi un TA 25 de TE) puisque toute l'amplification $\boldsymbol{A}_{2}$ se fait au niveau des étages d'entrée.

Pour réaliser l'opération $\Delta V / \Delta \theta$ qui fournit le pouvoir thermoélectrique $\alpha$, nous utilisons le même étage diviseur que précédemment (un inverseur double permet d'effectuer la commutation pour obtenir soit $1 / T$, soit $\alpha$ ). Nous nous sommes arrangés pour que la sortie $W$ du diviseur fournisse une tension représentative du pouvoir thermoélectrique telle qu'à $1 \mathrm{~V}$ corresponde $5000 / A_{2} \mu \mathrm{V} / \mathrm{K}$ (Fig. 8). L'étude des erreurs introduites par cet étage montre que l'on a intérêt à choisir $A_{2}$ aussi élevé que possible.

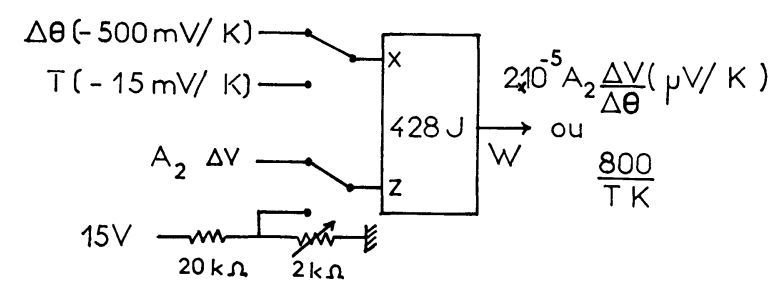

Fig. 8. - Etage diviseur.

3. Précision. - Notre étude a toujours été guidée par le souci d'obtenir les meilleures qualités du système et particulièrement la meilleure précision de chaque étage. Elle s'est constamment appuyée sur une évaluation aussi réaliste que possible des erreurs susceptibles d'affecter le traitement du signal.

3.1 PRÉCISION DES MESURES DE TEMPÉRATURES. L'erreur sur la mesure des températures provient principalement :

\section{- de l'amplificateur d'entrée}

- incertitude $\delta A_{1}$ sur l'ajustement du gain $A_{1}$;

- écarts dus aux dérives ;

\section{- du linéariseur}

- erreur systématique de linéarisation ;

- modification des caractéristiques des diodes avec les variations de la température ambiante.

Les erreurs introduites par les autres étages sont négligeables.

L'évaluation de ces différents termes conduit à admettre, pour une variation de $\pm 5^{\circ} \mathrm{C}$ de la température ambiante au cours d'une mesure, une erreur

$$
\varepsilon=1,9^{\circ} \mathrm{C},
$$

sur la mesure d'une température, et, une erreur relative

$$
\begin{array}{ll}
\pi=3 \% & \text { pour } \theta<-100^{\circ} \mathrm{C} \\
\pi=2 \% & \text { pour } \theta>-100^{\circ} \mathrm{C}
\end{array}
$$


sur la mesure d'une différence de températures supérieure à $0,5^{\circ} \mathrm{C}$.

D'autres facteurs sont susceptibles de diminuer la précision :

- Les capteurs : les mesures étant faites en régime quasi stationnaire l'erreur introduite par les capteurs provient essentiellement de l'écart entre la caractéristique des thermocouples utilisés et leur caractéristique standard, écart qui affecte surtout la mesure de la température moyenne. La norme NFE 18-001 prescrit les valeurs suivantes pour les tolérances sur les caractéristiques des thermocouples cuivre-constantan

$$
\begin{aligned}
& \pm 2 \% \mathrm{de}-100^{\circ} \mathrm{C} \text { à }-40^{\circ} \mathrm{C} \\
& \pm 0,8 \% \mathrm{de}-40^{\circ} \mathrm{C} \text { à }+100^{\circ} \mathrm{C} \\
& \pm 0,75 \% \mathrm{de}+100^{\circ} \mathrm{C} \text { à }+350^{\circ} \mathrm{C}
\end{aligned}
$$

- l'existence de forces électromotrices parasites d'origine thermoélectrique, au niveau du commutateur et dans la circuiterie des thermocouples.

Pour diminuer l'influence de ces forces électromotrices, difficiles à évaluer, il convient d'assurer un bon contact entre les thermocouples et l'échantillon, et de travailler avec des différences de température $\Delta \theta$ relativement élevées (de l'ordre de 5 à $15^{\circ} \mathrm{C}$ ).

3.2 PRÉCISION DES MESURES DE POUVOIR THERMOÉLECTRIQUE. - Dans les conditions d'ambiance précisées au paragraphe précédent, le diviseur fournit une tension

$$
\begin{aligned}
& W=\frac{10 A_{2}}{\Delta \theta} \times \\
& \quad \times\left(\Delta V \pm 12,5 \times 10^{-6} R \pm 36 \times 10^{-6} \pm \frac{53}{A_{2}} 10^{-2}\right)
\end{aligned}
$$

$R$ étant la résistance de l'échantillon exprimée en $\mathrm{M} \Omega$.

Cette relation tient compte des imperfections de l'amplificateur $A_{2}$ (courant d'entrée, dérive) et du diviseur (non-linéarité, dérive).

On peut ainsi considérer que la mesure de $\Delta V$ est affectée d'une erreur

$$
\delta(\Delta V)_{\mu \mathrm{V}}=12,5 R_{\mathrm{M} \Omega}+36+\frac{52,5}{A_{2}} 10^{4} .
$$

Cette erreur est assez importante et c'est essentiellement elle qui limite le champ d'utilisation de notre appareil.

En réalité cette limitation n'est pas aussi sévère que ne l'indique l'expression de $\delta(\Delta V)_{\mu \mathrm{v}}$. Dans la pratique, nous sommes très loin des différentes valeurs limites que nous nous sommes fixées pour évaluer la précision. Les expériences que nous avons effectuées ont montré que dans les conditions normales d'utilisation (différence de température de l'ordre de $10^{\circ} \mathrm{C}$ ), tension thermoélectrique suffisamment amplifiée $(>100 \mathrm{mV})$, l'erreur observée est inférieure à $5 \%$ et que l'appareil peut parfaitement mesurer des pouvoirs thermo- électriques de l'ordre de $10 \mu \mathrm{V} / \mathrm{K}$ sur des échantillons de résistance inférieure à $200 \mathrm{k} \Omega$.

Nous présentons, sur la figure 9, notre enregistrement du pouvoir thermoélectrique du nickel par rapport au cuivre (courbe $a$ ) et les résultats obtenus à partir des valeurs de Caskey [2] (courbe $b$ ). L'écart maximal ne dépasse pas $1,5 \mu \mathrm{V} / \mathrm{K}$.

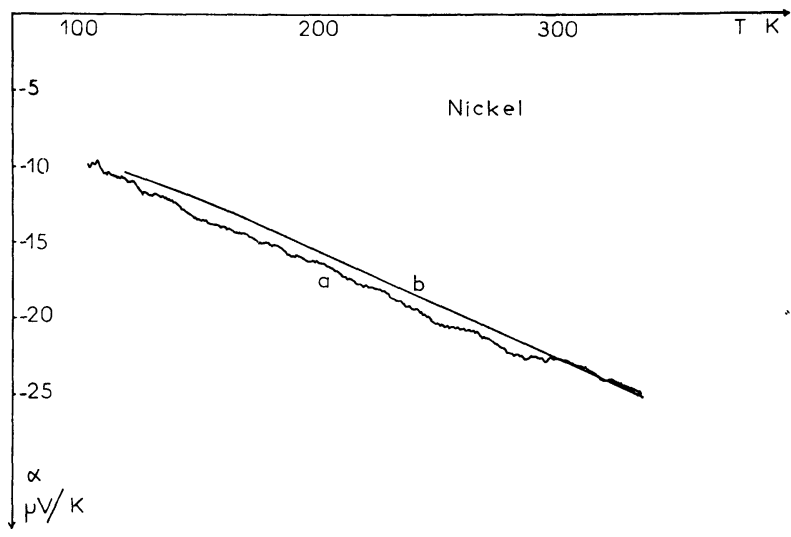

Fig. 9. - Pouvoir thermoélectrique du nickel par rapport au cuivre.

La figure 10 montre les résultats des mesures effectuées sur des échantillons en cours d'étude. Les croix + représentent des points expérimentaux obtenus par mesure directe de $\frac{\mathrm{d} V}{\mathrm{~d} \theta}$ en utilisant la méthode de Conan [1]. Signalons que cet auteur estime à $3 \%$ la précision de ses mesures sur des composés du type $\mathrm{MoTe}_{2-x}$ et $\mathrm{MoSe}_{2-x}$ [3].

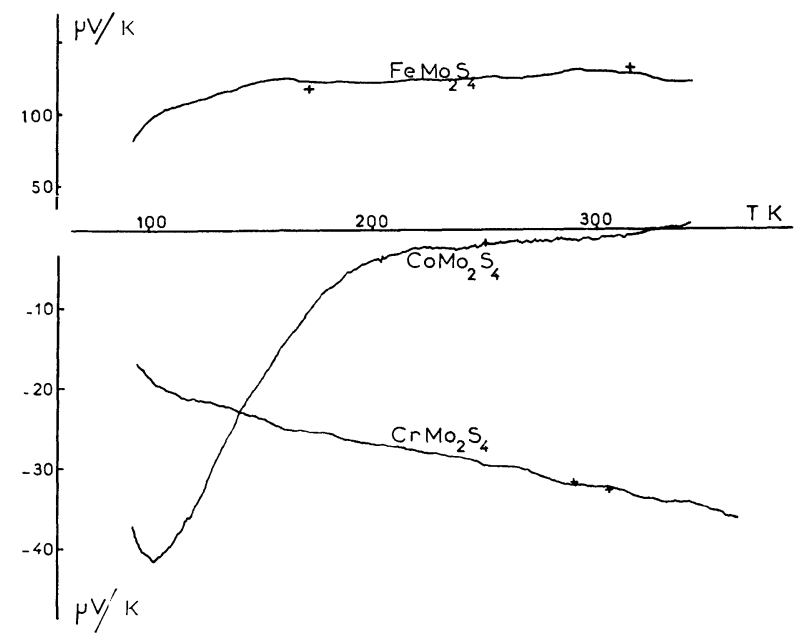

Fig. 10. - Pouvoir thermoélectrique des composés $\mathrm{MMo}_{2} \mathrm{~S}_{4}$ par rapport au cuivre.

4. Conclusion. - Nous avons réalisé un appareil permettant l'enregistrement direct du pouvoir thermo- 
électrique en fonction de la température. L'étude de la précision et les essais effectués ont montré que l'appareil construit avec des composants courants de bonne qualité, est suffisamment sûr pour permettre :

- d'obtenir la température moyenne d'un échantillon à moins de $1^{\circ}$ près et une différence de tempéra- ture à moins de $3 \%$ pour $\theta<170 \mathrm{~K}$, à moins de $2 \%$ pour $\theta>170 \mathrm{~K}$.

Nous l'avons largement utilisé en concurrence avec des méthodes de mesure point par point, et nous avons toujours constaté un excellent accord entre les résultats obtenus.

\section{Bibliographie}

[1] Conan, A., Thèse de Doctorat d'Etat, Nantes, 1972.

[2] Caskey, G. R., Sellmyer, D. J., Rubin, L. G., Rev. Sci. Instrum. 40 (1969) 1280.

[31 Conan, A., Goureaux, G., Zoaeter, M., J. Phys. Chem. Sol. 36 (1975) 315. 